\title{
Anti Crosstalk Ranging Method with Multi Ultrasonic Sensor Based On Chaotic Sequence
}

\author{
Anhua Wang ${ }^{1,2}$ and Yunbo Shi ${ }^{1 *}$ \\ ${ }^{1}$ The Higher Educational Key Laboratory for Measuring \& Control Technology \\ and Instrumentation of Heilongjiang Province, Institute of Measurement-Control \\ Technology \& Communications Engineering, Harbin University of Science and \\ Technology, Harbin, China, 150080. \\ ${ }^{2}$ School of Electrical \& Control Engineering, Heilongjiang University of Science \\ and Technology, Harbin, China, 150022 \\ 3036361_cn@sina.com,shiyunbo@126.com
}

\begin{abstract}
Ultrasonic sensor is widely used in robot, automobile, automatic production line and so on, but its detection angle is limited, therefore, multiple ultrasonic sensors are often needed to expand the detection range of distributed structures such as parallel or surround. Multiple ultrasonic probes work at the same time, and very close each other, it will cause crosstalk problems. The chaotic sequence is introduced into the detection signal in the system of ultrasonic receiving and transmitting, it can improve the reliability of detection information and efficiency, realize multi probe synchronously work without crosstalk. Simulation and experimental data show that the system can effectively detect in the range from 0.08 to 4.87 meters, the average error is less than $1.16 \%$, which proves that this method is feasible.
\end{abstract}

Keyword: Ultrasonic sensor, Distance measurement, Chaotic sequence, Anti crosstalk

\section{Introduction}

Distance measuring device of ultrasonic has advantages such as high integration, High cost performance, Small volume, and high anti-interference, it is suitable for short distance measurement, and it is applied to industrial robot, mechanical processing, navigation system, etc. Ultrasonic ranging technology developed from the end of the 20th century, HanneSElmer blend of coding technology with ultrasonic distance measurement in 2007, it improves the measurement resolution. APREsys company of America present their height measuring instruments in 2008. In the $1950 \mathrm{~s}$, some ultrasonic instrument were introduced from oversea to china, with the continuous expansion of social needs and the support of the state, more and more people began to research and application of ultrasonic detection technology[1]. Zhang Song designed the ultrasonic ranging system based on $m$ sequence [2]. M sequence is spread spectrum signal, the frequency spectrum of the signal which transmitted in communication system has been broadening, and the frequency spectral density is very low, so the spread spectrum communication system has potential ability of anti frequency selective fading. Most of the pseudo-random sequence used to spread spectrum are generated by shift register, such as m sequence, GOLD sequence. However, these sequences have the periodic characteristic, and circuit structure is also more complex. With the rapid development of communication technology, these sequences are increasingly unable to meet the requirements of communication [3]. A method of synchronous distance measurement with large angle and non crosstalk multi ultrasonic sensor was introduced in this paper, which is applied to the work of positioning the wounded. 


\section{Ultrasonic Ranging Method}

\subsection{Principle of Distance Measurement}

According to the principle of distance measurement, methods of ultrasonic distance measurement include phase measurement, amplitude measurement and transit time measurement. The first method is noted of its high detecting precision, but its calculation is complicated; the strength of the second method is circuit compact, but its detecting precision and anti - jamming ability is bad; the last method easy to achieve, it owned many virtues: high ranging accuracy, better anti-interference, and low cost. According to the demand of the rescue robot, the method of transit time measurement is selected.

The method of transit time measurement is simple. The time of the ultrasound wave transferred is detected from transmitter to receivers, this time is the transit time, and then calculate the distance according to the speed of ultrasonic wave. The measure principle is shown in Figure 1. $\mathrm{L}$ is the distance to be measured, $\mathrm{d}$ is the true distance, the angle $\alpha$ is negligible.

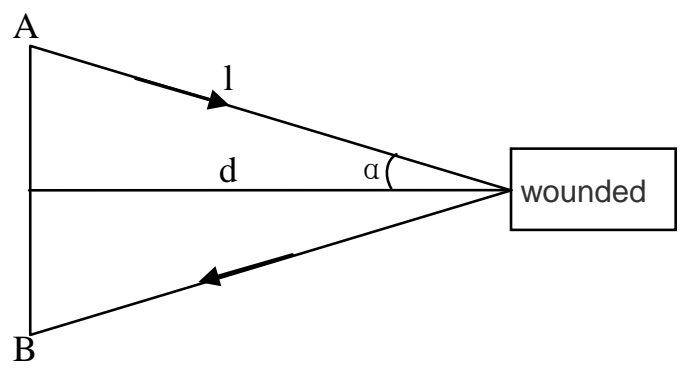

Figure 1. Principle of Distance Measurement

Specific steps are as follows:

(1) The chaotic sequence is generated by the signal circuit and transmitted to the probe;

(2) Probe transmits chaotic sequence signal, and at the same time the timer is started;

(3) After short delay, the receiver receives the reflected wave and judges it by calculating correlation, if it in accord with the transmitted wave, then stops the timer;

(4) According to the timing results, compute transit time t, according to the wave velocity of the ambient temperature, compute the distance of the target.

If $\mathrm{T} 1$ is the overflow cycle of timer, $\mathrm{n}$ is the number of overflow interrupt, and $\mathrm{T} 2$ is the stored value of count register when timer is stop, $\mathrm{f}$ is working frequency, the transit time expression is:

$$
t=\frac{n T 1+T 2}{f}
$$

As shown in Figure 1, 1 represent the distance, $v$ is the ultrasonic speed, then:

$$
l=\frac{v t}{2}=\frac{v(n T 1+T 2)}{2 f}
$$

\subsection{Method of Producing Chaotic Sequence}

2.2.1. Selection of Chaotic Sequences: Chaos is a special kind of dynamic system, it can provide a large number of signals, which are unrelated, random, determined, and easy to generate and regenerate. The chaotic sequence can be generated as long as a mapping formula and a initial value, and don't need store the value of the each sequence points. According to the characteristics of chaos, if the chaotic sequence is used to replace the general pseudo random sequence, it not only provides the conditions for communicating between multi users, but also can realize the encryption of information[4]. Therefore, the 
Logistic chaotic sequence is used as the detection signal for the distance measurement method, which is introduced in this paper, by the characteristics of chaotic sequence, such as the strong sensitivity of initial value and good self correlation, the multi probe synchronization measurement is realized.

2.2.2. Generation of Logistic Chaotic Sequences: Logistic mapping is also called insect mouth mapping, it was first put forward by Pierre Verhulst when he was researching the question about quantity of insect population, it belongs to one dimensional discrete model of chaotic, mathematical expressions is:

$$
x_{n+1}=\mu x_{n}\left(1-x_{n}\right) \quad 0<x_{n}<1
$$

In the formula (3), $x_{n}$ is state parameter, $\mu$ is branch parameter, if $3.5699465<\mu \leq 4$, this mapping will be in a state of chaos. A number of different chaotic sequences will be generated at the same time by changing initial value and parameter of the system.

The digital circuit of the system is shown in Figure 2, set the initial value of the circuit, Constant $=0.15$, the high level of the pulse is used as the initial input of the data selector; When the pulse signal is turned into a low level, system begin to iterate, output signal is generated through delayer, multiplier, adder and amplifier, then realize the interval quantization work by the barrel shift register and bit sampling device. Quantization function is:

$$
T\left[x_{n}\right]=\left\{\begin{array}{l}
1, \quad x_{n} \in U_{k=0}^{2^{m}-1} I_{2 k+1}^{m} ; k=0,1,2 \ldots \\
0, x_{n} \in U_{k=0}^{2^{m}-1} I_{2 k}^{m}
\end{array} ; k\right.
$$

In the formula (4), $m$ is an integer greater than zero, $I_{0}^{m}, I_{1}^{m}, I_{2}^{m}, \ldots$ is continuous and equal sub interval in the interval $[0,1]$, when the interval consists of an odd number as the initial, and the chaotic signal just in this interval, the result of quantization is 1 ; when the interval consists of an even number as the initial, and the chaotic signal just in this interval, the result of quantization is 0 .

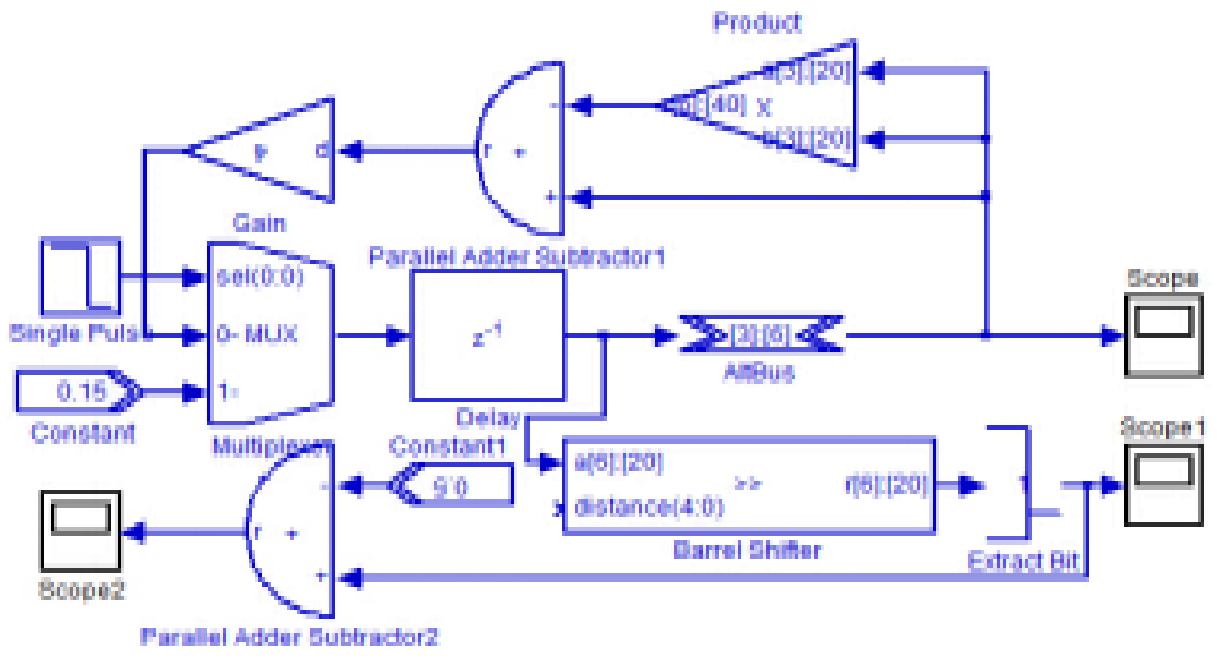

Figure 2. Generation Circuit of Logistic Chaotic Sequence 


\section{Hardware Design of Ranging System}

The ranging system is composed of a signal generating circuit, a control circuit, a transmitting circuit, a receiving circuit, a signal conditioning, a comparison circuit and a display circuit. As shown in Figure 3, chaotic sequence is generated by the signal generator circuit, and is stored to the core controller, then the signal is transmitted to the ultrasonic transmitter by the transmitting circuit, set the timer going at the same time; after receiving the signal at the receiving end, the signal is processed by conditioning circuit, and it is judged whether it is a valid signal, if the signal is valid, then stop timing, at the same time, performing the work of calculation and display.

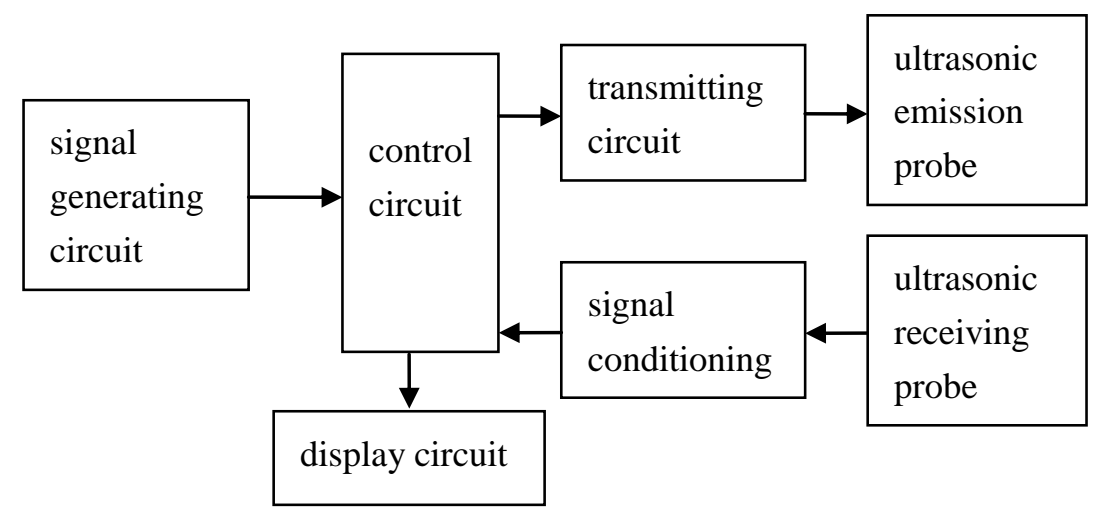

Figure 3. Hardware Structure of System

The chaotic sequence is generated by FPGA, it is transmitted to transmitting circuit passing by controller, and the transmitting circuit converts the chaotic sequence to the signal which is fit to be transmitted by the ultrasonic probe. After the signal arrives at the receiving module, it will be amplified and filtered, the characteristic curve of correlation between receiving and transmitting signals is obtained by the controller, if the receiving wave and the transmitted wave are consistent, the expected peak will appear in the characteristic curve, and finally the controller will calculate the distance value. Signal transmitter, receiver and conditioning circuit as shown in Figure 4.

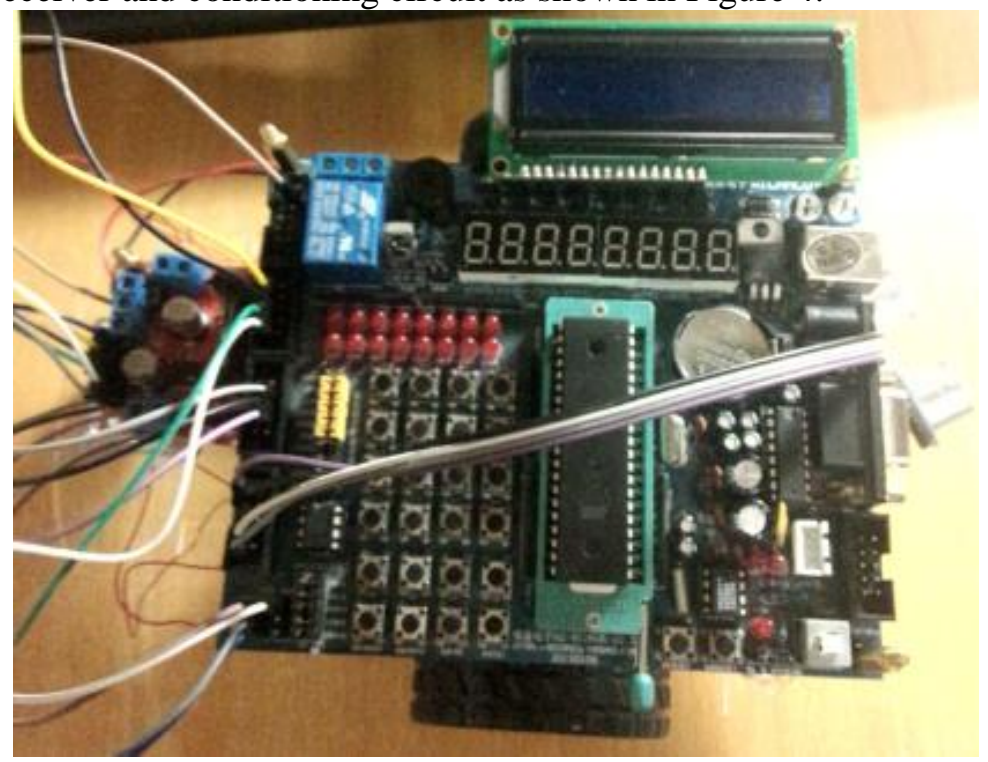

Figure 4. Transmitting Circuit, Receiving Circuit and Conditioning Circuit 


\section{Analysis of Experimental Result}

\section{1, Simulation of Logistic Chaotic Sequence Characteristic}

The Simulink simulation model of Logistic chaotic sequence is established applying the DSP Builder toolbox in MATLAB, and according to the formula (3) (4). Adjust parameters to obtain a variety of characteristic waveforms of Logistic chaotic sequence. Prove the feasibility of the method introduced in this paper. At same time convert the model to VHDL and TCL, in order to shorten the hardware design cycle of FPGA.

4.1.1. Initial Value Sensitivity of Logistic Chaotic Sequence: The experimental waveforms of initial value sensitivity are shown in Figure 5, use two very similar number as the initial value of the system, enter into iteration many times. Visible, the two waves finally completely separated, meet the characteristic of the huge difference in the outputs with two very close initial values, it is proved that the initial value sensitivity of Logistic chaotic sequence is strong, and multi-channel detection signal easy to realize by Logistic chaotic sequence.

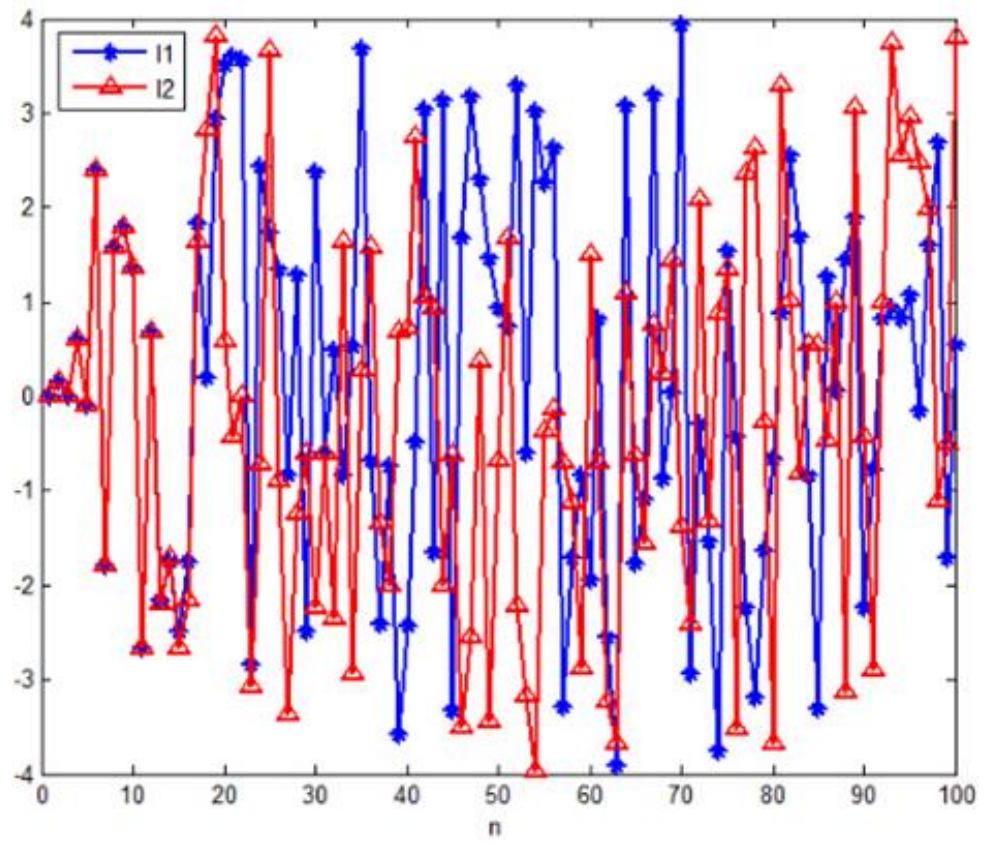

Figure 5. Initial Value Sensitivity of Logistic Chaotic Sequence

4.1.2. Random Characteristic of Logistic Chaotic Sequence: Random characteristic is an important performance of chaotic sequence. In order to study the influence of parameter setting on the randomness of chaotic sequence, change the parameter $(m: n)$ of AltBus module in the generation circuit of chaotic signal, analyze the random characteristic of Logistic chaotic sequence by the autocorrelation function and the waveform of output. AltBus (m:n) module can complete the transformation from floating-point operation to fixed-point arithmetic, $m$ is the Figures of binary on the left of the decimal point, $\mathrm{n}$ is the Figures of binary on the right of the decimal point [5] . The data type conversion can be completed by the AltBus module, so the circuit can be realized in hardware.

The time domain waveform of chaotic signal is shown in Figure 6. Fix the parameter $m$ which belongs to (m:n) of AltBus module, when $n$ is small, the waveform is periodic, while the $\mathrm{n}$ turn larger, the waveform is pseudo random and bounded, it prove that the parameter of AltBus module (m:n) effect on the randomness of chaotic signals. 

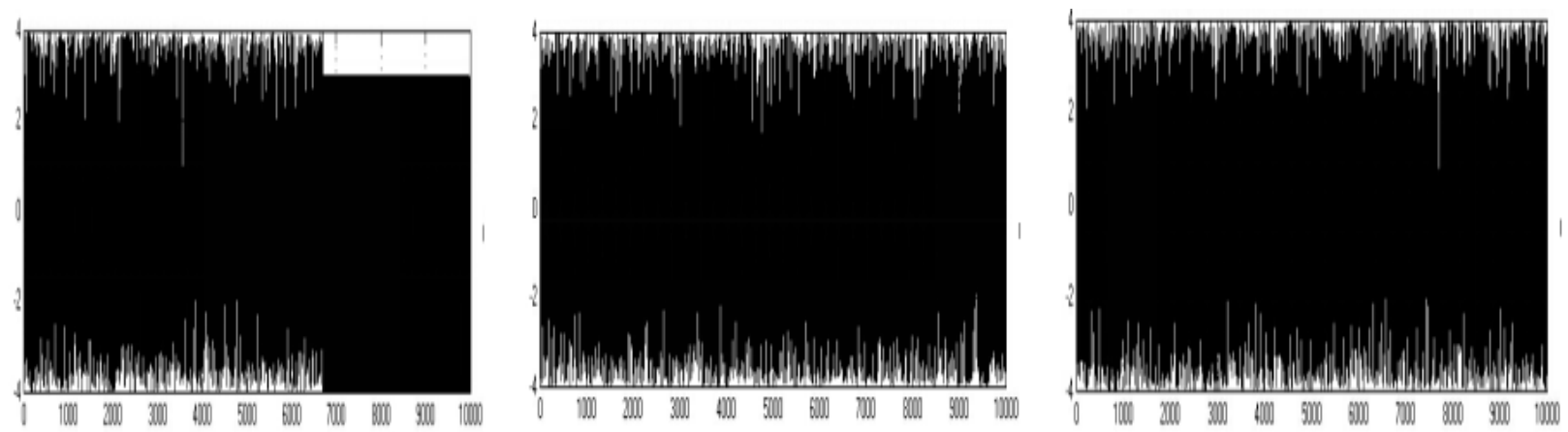

Figure 6. The Time Domain Waveform of Chaotic Signal

The autocorrelation characteristic of chaotic signal is shown in Figure 7. Fix the parameter $\mathrm{m}$ which belongs to (m:n) of AltBus module, with the increase of $\mathrm{n}$, the self correlation of Logistic chaotic signal turn to good and stable. It is proved that chaotic sequences with different precision and different random characteristics can be obtained by setting the parameters of AltBus module (m:n), and easy to generate multiple synchronous detection signal.

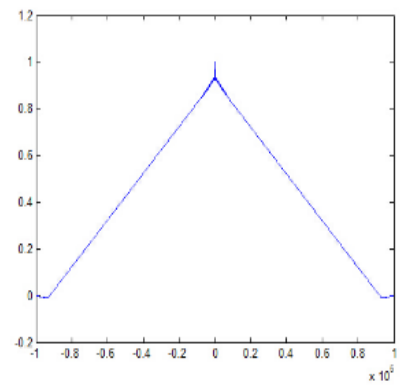

$\operatorname{AltBus}(3: 6)$

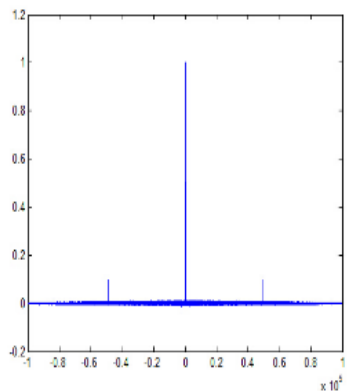

$\operatorname{AltBus}(3: 8)$

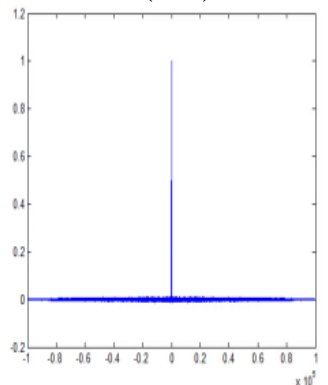

AltBus(3:9)

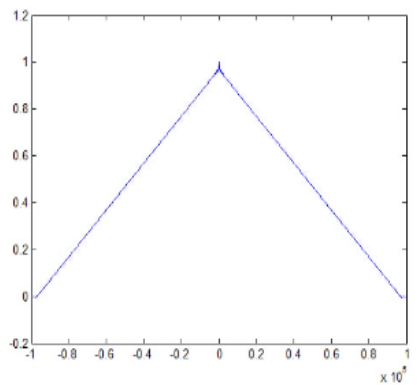

AltBus(4:6)

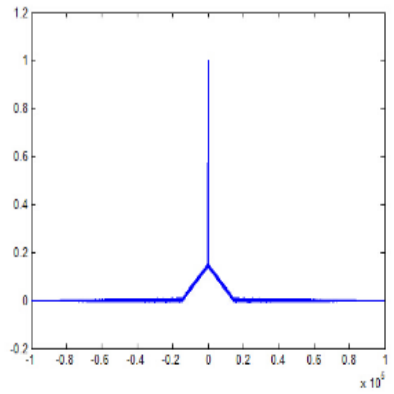

AltBus(4:8)

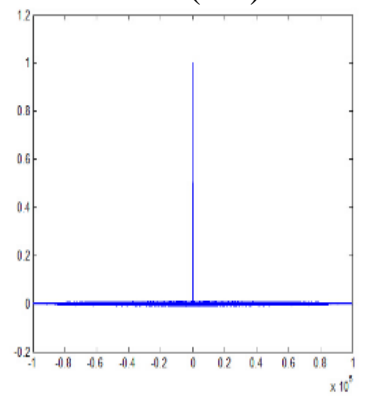

AltBus(4:9)

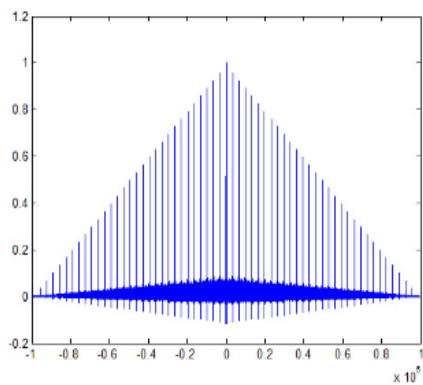

$\operatorname{AltBus(5:6)}$

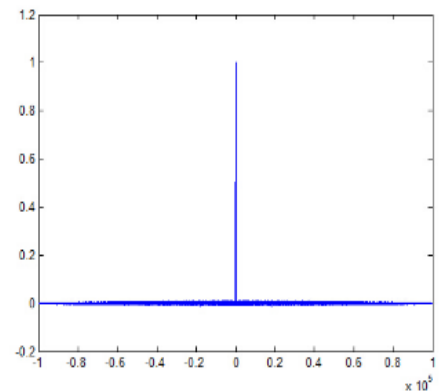

AltBus(5:8)

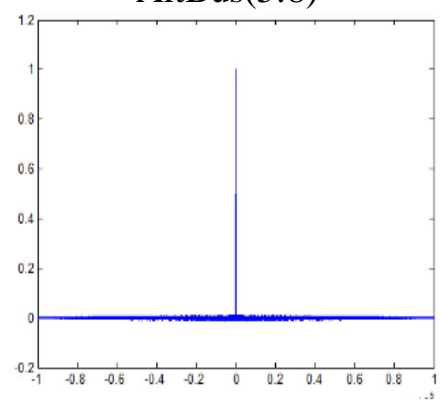

AltBus(5:9) 


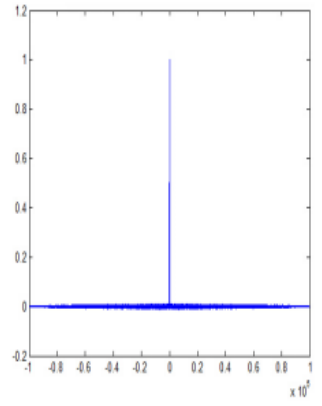

$\operatorname{AltBus}(3: 15)$

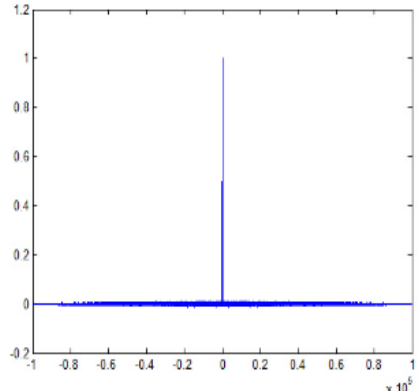

AltBus(4:15)

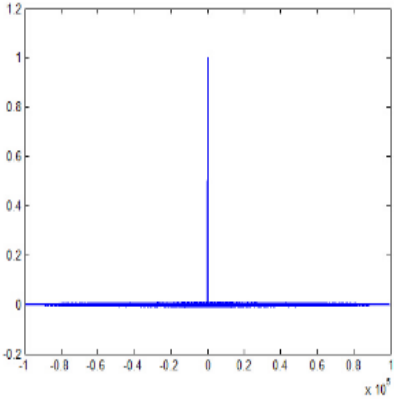

$\operatorname{AltBus}(5: 15)$

Figure 7. The Autocorrelation Characteristic of Chaotic Signal

\subsection{Experiment}

In the laboratory, experiments were made on the device of ultrasonic distance measurement which is introduced in this paper, there is no interference between the probe, the relationship between distance and error is obtained, as shown in Table 1. The maximum detection range of this device is 4.87 meters, in the range from 0.3 meters to 4.6 meters; the average error is less than $1.16 \%$.

Table 1. Test Data

\begin{tabular}{l|l|l|l|l|l|l|l|l}
\hline test value & 0.067 & 0.292 & 0.589 & 0.994 & 1.302 & 1.606 & 2.030 & 2.295 \\
\hline actual value & 0.080 & 0.300 & 0.600 & 1.000 & 1.300 & 1.600 & 2.000 & 2.300 \\
\hline absolute error & -0.013 & -0.008 & -0.011 & -0.006 & 0.002 & 0.006 & 0.030 & -0.005 \\
\hline relative error & $19.4 \%$ & $2.7 \%$ & $1.9 \%$ & $0.6 \%$ & $0.2 \%$ & $0.4 \%$ & $1.5 \%$ & $0.2 \%$ \\
\hline
\end{tabular}

\begin{tabular}{l|l|l|l|l|l|l|l|l}
\hline test value & 2.614 & 3.043 & 3.303 & 3.618 & 4.105 & 4.221 & 4.519 & 4.704 \\
\hline actual value & 2.600 & 3.000 & 3.300 & 3.600 & 4.000 & 4.300 & 4.600 & 4.870 \\
\hline absolute error & 0.014 & 0.043 & 0.003 & 0.018 & 0.105 & -0.079 & -0.081 & -0.166 \\
\hline relative error & $0.5 \%$ & $1.4 \%$ & $0.1 \%$ & $0.5 \%$ & $2.6 \%$ & $1.9 \%$ & $1.8 \%$ & $3.5 \%$ \\
\hline
\end{tabular}

\section{Conclusions}

In this paper, a large range of distance measuring device composed of 3 ultrasonic sensors is introduced. The random characteristic of chaotic sequence is obtained by simulation, it is proved that the introduction of chaotic sequence is a feasible to eliminate the crosstalk between probes; Test data indicates that the effective range of detection is $0.3 \sim 4.6$ meters, the average error is less than $1.16 \%$, this method can realize multi probe synchronously work without crosstalk.

\section{Acknowledgment}

This work is partially supported by National Nature Science Fund (51304075) "Research of Intelligent control method of cantilever type unmanned roadheader section forming based on strapdown inertial navigation system".

\section{References}

[1] Y. Li, "Ultrasonic distance measurement system based on HT46F49E", Chang'an University, (2011), vol. 6. pp. 2-3.

[2] S. Zhang and Y. Zhang, "Non crosstalk ultrasonic distance measurement system based on m sequence", Electronic measurement technology, (2015), vol. 9. pp. 19-24.

[3] L. Wang, F. Gong and C. Yang, "Study on the performance of Chebyshev chaotic sequences", User of instrument and meter, (2008), vol. 2. pp. 94-96. 
International Journal of Future Generation Communication and Networking

Vol. 9, No. 11 (2016)

[4] J. Wang, Y. Wang and C. Yin, "Investigation on the Simulation of One-Dimensional Discrete Chaotic Digital Generation Circuit”, RVSP2015, vol. 11, (2015), pp. 180-184.

[5] J. Wang, "The Simulation Research and NIST Test of Binary Quantification Methods forthe Typical Chaos", RVSP2015, vol. 11, (2015), pp. 195-199. 